\title{
Triglyeride-induced Pancreatitis: Diagnostic and Therapeutic Approach
}

\author{
Marvin Louis Roy Y. Lu" ${ }^{1 *}$ and Markus D. Agito \\ ${ }^{1}$ Albert Einstein Medical Center, Department of Internal Medicine, Philadelphia, PA, USA \\ ${ }^{2}$ University of Florida, Division of Gastroenterology, Hepatology and Nutrition, Gainesville, FL, USA
}

\begin{abstract}
Hypertriglyceride-induced pancreatitis is rarely associated with triglyceride levels less than $1000 \mathrm{mg} / \mathrm{dl}$. However, triglyceride levels can drop rapidly with fasting that often accompanies acute pancreatitis. In addition, triglyceride levels can be elevated in the setting of acute pancreatitis as injury to the pancreas causes inadequate lipid metabolism leading to triglyceride elevation. Thus, the question arises whether high triglycerides induce acute pancreatitis or vice versa in patients presenting with elevated triglyceride levels. Also, the drop in triglyceride levels associated with fasting may result in failure to consider hypertriglyceridemia as the etiology for acute pancreatitis in a patient who presents with triglyceride levels below $1000 \mathrm{mg} / \mathrm{dL}$. In this review, we discuss the relationship between hypertriglyceridemia and pancreatitis as well as causes of elevated triglyceride levels that must be considered in patients who present with both conditions. In addition, we present an evidence based algorithm that summarizes the approach to hypertriglyceridemia and pancreatitis in the setting of complex medical co-morbidities.
\end{abstract}

\section{Introduction}

Hypertriglyceride-induced pancreatitis is the third most common cause of pancreatitis after gallstones and alcohol [1,2]. It accounts for 1.3 to 3.8 percent of pancreatitis cases [1] and in one study, it was the identified etiology in $77.8 \%$ of severe acute pancreatitis in pregnancy with a mean triglyceride level at onset of $27.9 \mathrm{mmol} / \mathrm{l}$ [3]. Pancreatitis usually develops in patients with markedly elevated serum triglyceride levels (>1000 mg/dL) and/or lactescent serum specimens [1] which has been associated with increased mortality compared to other causes of pancreatitis [4]. Since measurement of serum triglyceride levels is often not considered at the time of admission in the setting of acute pancreatitis, failure to identify an obvious yet lethal cause may be missed easily because triglyceride levels rapidly drop during voluntary fasting [5]. There are no studies in the literature ascertaining how many of hypertriglyceride-induced pancreatitis cases are missed initially. The purpose of this review article is to elucidate an evidenced based algorithm to correctly identify these patients.

\section{Demographics}

In one large retrospective cohort study, patients who presented with hypertriglyceridemia-induced pancreatitis had a mean age of $54.2 \pm 11.9$ years. Three fourths of which are male and a great majority (78.9\%) are Caucasians [6].

\section{Clinical presentation}

The initial presentation of Hypertriglyceridemia-induced pancreatitis is the same as that of pancreatitis of other causes (Table 1). It should be included as part of the differentials in any patient presenting with signs and symptoms of pancreatitis with a history of poorly controlled diabetes, hyperlipidemia or a family history of hyperlipidemia, hypothyroidism, alcoholism, and pregnancy $[1,2,7,8]$. The prevalence of diabetes among patients with hypertriglyceridemia is $41 \%$, with an additional $11.6 \%$ having impaired glucose metabolism.
In addition, all patients with hypertriglyceridemia have at least one secondary factor contributing to their hypertriglyceridemia [6].

Severe clinical clues on physical examination may help physicians to suspect hypertriglyceridemia. Xanthomatas are localized infiltrates of lipid-containing foam cells which may be found within the skin, tendons and subperiosteum in patients with hypertriglyceridemia. Xanthomas in the dorsum of hand and ankle (heterozygous familial hypercholesterolemia), planar xanthomas in the antecubital fossa (homozygous familial hypercholesterolaemia), xanthomata on the palms, elbow and extensor surface of arms (type III hyperlipoproteinaemia) and eruptive xanthomata on the extensor surfaces of the forearm, lipemia retinalis, hepatosplenomegaly and milky plasma samples are all indicative of severe and chronic hypertriglyceridemia [9]. These classical findings however, have a very low incidence and are found in only less than $10 \%$ of cases. In addition, the triglyceride levels do not accurately predict whether patients would develop these signs, suggesting that factors other than

Table 1. Diagnosis of acute pancreatitis (2 out of 3) [15].

Abdominal pain characteristic of acute pancreatitis a

Serum amylase and/or lipase $\geq 3$ times the upper limit of normal

characteristic findings of acute pancreatitis on CT scan $b$

a - Unbearable epigastric pain radiating to the back in half of the cases, reaching max intensity in 30 minutes and persists for more than 24 hours without relief

b - Enlargement of the pancreas with diffuse edema, heterogeneity of pancreatic parenchyma, peripancreatic stranding, and peripancreatic fluid collections

Correspondence to: Dr. Marvin Louis Roy Lu, Albert Einstein Medical Center, Department of Medicine, 5501 Old York Road, Philadelphia, USA, Tel: (215) 456 8520; E-mail: lumarvin@einstein.edu

Key words: acute pancreatitis, hypertriglyceridemia, algorithm, hypertriglyceride induced pancreatitis

Received: March 10, 2016; Accepted: April 15, 2016; Published: April 20, 2016 
hypertriglyceridemia may contribute to their development [6].

Hypertriglyceridemia induced pancreatitis usually occurs when the serum triglyceride is more than $20 \mathrm{mM}(1772 \mathrm{md} / \mathrm{dl})$ acutely [6].

Serum amylase levels may appear falsely normal in patients with pancreatitis and serum triglyceride levels ranging from 500 to 6000 $\mathrm{mg} / 100 \mathrm{~mL}$. This was first thought to be due to interference of light transmission in the colorimetric reaction caused by lactescent serum specimens [10]. However, Warshaw et al. suggested the presence of irculating amylase inhibitors in the serum and urine in patients with hypertriglyceride-induced pancreatitis [11]. True amylase levels can be determined after correcting the lactescent serum with serial dilutions with normal saline $[10,11]$. Interestingly, in one animal study, amylase levels were found to decrease by a factor of 4.33 in pancreases from hyperlipidemic acute necrotic pancreatitis compared with the normallipid acute necrotic pancreatitis group rats. It was suggested that the high-fat low-carbohydrate diet given to the hyperlipidemic rats may suppress the need for amylase and thus its mRNA expression in the pancreas [12].

\section{Pathogenesis}

There are a number of proposed mechanisms by which hypertriglyceridemia can cause pancreatitis. It may be secondary to rapid accumulation of chylomicrons in the pancreatic vasculature [13]. Chylomicrons are the largest lipid-transporting lipoprotein and can form a thrombus plug and obstruct the pancreatic circulation during rapid buildup causing ischemia and acidosis to the organ [13]. The acidic environment may cause premature activation of trypsinogen leading to autodigestion. Another hypothesis is that it may be secondary to the release of free fatty acids from triglycerides by pancreatic lipase [14]. These pro-inflammatory free fatty acids can induce free radical formation and damage to the pancreas or may in fact cause direct injury through chemical irritation together with lisolecithin [15]. Free fatty acids were found to cause edema and hemorrhage of canine pancreatic glands by Saharia et al. [14]. Hyperlipidemia induced pancreatitis in pregnancy is believed to be secondary to acute adipose infiltration and fat embolism of pancreatic vessels as a result of fat dissociation by human placental lactogen from the syncytiotophoblasts and release of a substantial aggregate of free fatty acids [15].

\section{Diagnostic approach}

Patients with severe hypertriglyceridemia causing pancreatitis should be screened for the following:

1. Familial causes such as Type I, IV and V hyperlipidemia syndromes

2. Alcohol, Thyroid stimulating hormone (TSH), and Glucose levels

3. Medications that can cause hypertriglyceridemia such as steroids and valproic acid.

\section{Familial hyperlipidemia syndromes}

Type I and V causes severe hypertriglyceridemia enough to cause spontaneous pancreatitis while Type IV usually requires a secondary factor such as alcohol, diabetes mellitus, obesity or thyroid dysfunction to cause the disease [16].

Type I hyperlipoproteinemia is a rare genetic disorder of autosomal recesive trait. It can be classified further based on its defect: 1a lipoprotein lipase deficiency, $1 \mathrm{~b}$ - apoprotein II deficiency; and 1c - presence of a lipoprotein lipase inhibitor. All defects cause an increase in circulating chylomicrons and subsequent hypertriglyceridemia. The resulting accumulation of triglycerides causes the patient's plasma to have a lactescent appearance. Patients with this disorder usually presents in childhood with eruptive and tuberous xanthomas and may have recurrent pancreatitis [16]. Familial lipoprotein lipase deficiency (1a) can be diagnosed by LPL enzyme assay. Management of the disorder includes maintaining a daily fat intake of $20 \mathrm{~g}$ or less or $15 \%$ of total caloric intake. In addition, patients should avoid medications known to cause increased levels of triglycerides such as ethanol, estrogens, diuretics, and beta blockers [17]. In a 2009 study published in BMC Gastroenterology comparing twenty-four survivors of acute pancreatitis secondary to hypertriglyceridemia to a control of thirty one patients with severe hypertriglyceridemia who had not developed pancreatitis, five cases were found to have lipoprotein lipase deficiency [18]. There were no differences between the groups in terms of other risk factors for pancreatitis such as obesity, diabetes, or alcohol abuse outside of the acute phase. All of the patients found to have lipoprotein lipase (LPL) deficiency were thin and experienced their first episode of pancreatitis in childhood. The authors recommend measurement of lipoprotein lipase activity be reserved for patients who experienced their first episode of acute pancreatitis in childhood [18].

Type $\mathrm{V}$ hyperlipoproteinemia is characterized by an increase in both chylomicrons and very low density lipoprotein (VLDL). Patients with this disorder often have low low-density lipoprotein (LDL) levels as well. Such patients have been found to demonstrate particularly labile triglyceride levels with sharp increases when consuming alcohol and sharp decreases when eventually ceasing alcohol use [20]. These triglyceride fluctuations may delay the consideration of the lipid disorder as a possible factor contributing to acute pancreatitis. However, the disorder should be considered in all patients who have triglyceride levels greater than $1000 \mathrm{mg} / \mathrm{dL}$ at any point of time in their care. Type $V$ hyperlipoproteinemia can be seen in patients with alcohol or diabetes induced hypertriglyceridemia. These factors simultaneously contribute to the development of pancreatitis. In these patients, the genetic disorder contributes to the overproduction of VLDL and chylomicrons, while alcohol or diabetes interferes with the clearance of triglycerides [9]. Estrogen therapy has also been found to induce pancreatitis in women with type V hyperlipoproteinemia [19].

Type IV hyperlipoproteinemia is an autosomal dominant condition characterized by an increased production of VLDL and generally require another factor such as diabetes or alcohol in order to induce pancreatitis $[9,21]$. Patients with this disorder typically have triglyceride levels below $1000 \mathrm{mg} / \mathrm{dL}$. Therefore, this condition should generally be considered in the presence of contributing factors.

\section{Secondary causes of hypertriglyceridemia}

Non-insulin dependent diabetes mellitus is a well-known cause of hypertriglyceridemia and is themost common cause of hypertriglycerideinduced pancreatitis [1]. There are several mechanisms that may explain this phenomenon. One described thoroughly in literature that may play the largest role in hypertriglyceridemia in insulin resistant patients is the loss of the inhibitory effect of insulin on VLDL apolipoprotein $\mathrm{B}$ production by hepatocytes [22]. In addition, Lipoprotein lipase is regulated by insulin levels through gene expression, synthesis and secretion. All of these factors may be impaired in insulin-resistant patients [23]. While hypertriglyceridemia is the most common lipid abnormality in these patients, most well controlled diabetics have triglyceride levels that are within the normal range [24]. However, 
abrupt elevations in serum triglyceride levels can occur and may result in pancreatitis [24].

Like triglyceride induced pancreatitis, patients with diabetic ketoacidosis (DKA) may also present with markedly elevated serum triglycerides and acute abdominal pain making it more difficult to differentiate the two entities [16,25]. Deficiency of insulin promotes lipolysis in adipose tissue followed by release of free fatty acids which will eventually be converted to very low density lipoproteins, which, coupled with the inhibition of lipoprotein lipase in peripheral tissues, results in hypertriglyceridemia [26]. Nair et al. evaluated the incidence of hypertriglyceridemia and occult pancreatitis in 100 patients with diabetic ketoacidosis. Their study showed that patients with DKA presenting with acute abdominal pain and hypertriglyceridemia $(>500$ $\mathrm{mg} / \mathrm{dl}$ ) may also have CT scan findings consistent for pancreatitis. DKA patients with pancreatitis had higher glucose levels with higher anion gap acidosis compared to the patients without pancreatitis. Serum triglyceride levels improved with resolution of ketosis but not with acidosis or anion gap [16]. The degree of hypertriglyceridemia however, did not correlate with the severity of ketoacidosis [25].

Alcohol, when taken with a meal high in saturated fat, can increase postprandial triglyceride level significantly. This is mainly because of alcohol's inhibitory effect on lipoprotein lipase, thereby decreasing the breakdown of chylomicrons and VLDL [26]. On the other hand, excessive alcohol intake can cause hypertriglyceridemia even in a fasting state because it promotes the synthesis of large VLDL particles in the liver. Furthermore, alcohol increases the synthesis of large VLDL particles in the liver, which is the main source of triglycerides in the hypertriglyceridemia associated with chronic excessive alcohol intake. In case of chronic consumption, lipoprotein lipase activity seems to adapt itself. [26]. In an animal study comparing the triglyceride levels after acute and chronic ingestion of alcohol, it was shown that the increase in triglyceride levels were less pronounced in rats subjected to chronic alcohol intake in a non-fasting state, confirming that chronic alcohol intake stimulates the production of extrahepatic lipoprotein lipase in response to the increased triglyceride concentration [12]. In some cases, acute alcohol intake may cause significantly elevated triglyceride levels with an increased risk of pancreatitis, especially patients with metabolic syndrome [26].

Medications such as estrogen, estradiol, glucocorticoids, thiazide diuretics, beta blockers, sertraline, protease inhibitors, valproate and related drugs, and isotretinoin can cause severe hypertriglyceridemia and the chylomicronemia syndrome in patients with inherited lipid metabolic syndromes (Table 2) [27]. These drugs reduce lipoprotein lipase and hepatic triglyceride lipase activity [28]. Oddly, fenofibrates, which decrease triglyceride levels, causes an increased risk of pancreatitis among patients with type 2 DM [29].

Table 2 shows a list of genetic and acquired cause of severe hypertriglyceridemia that maybe associated with pancreatitis. This was adopted from a scientific statement by the American Heart Association on triglycerides and cardiovascular disease [30].

\section{Initial treatment}

Initial treatment of hypertriglyceride-induced pancreatitis is no different from treating other causes of pancreatitis (bowel rest, aggressive intravenous hydration, pain control and anti-emetics). A recent study shows that goal-directed hemodynamic management guided by functional hemodynamic parameters such as stroke volume variation, compared to CVP-guided therapy, led to a significantly
Table 2. Causes of Sever Hypertriglyceridemia that maybe associated with pancreatitis [40].

\section{Genetic}

Lipoprotein Lipase Deficiency (Type 1a)

Apolipoprotein CII Deficiency (Type 1b)

Apolipoprotein AV deficiency

Glycosylphosphatidylinositol-anchored HDL binding protein-1 (GPIHBP1) Deficiency

Marinesco-Sjogren Syndrome

Chylomicron retention (Anderson) disease

Familial hypertriglyceridemia (Type IV)

Acquired Disorders of Metabolism*

Poorly controlled insulinopenic diabetes

Hypothyroidism

Pregnancy, especially in the 3rd trimester +

Drugs

a-interferon

Atypical antipsychotics

Table

Beta blockers such as atenolol $\Psi$

Bile acid resins

L-Asparaginase

Estrogens (oral, not transcutaneous)

Protease inhibitors

Raloxifene $\Delta$

Retinoic acid drugs

Sirolimus

Tamoxifen

Thiazides

Diet

Alcohol Excess, especially with ah high saturated-fat diet

Diseases

Autoimmune Chylomicronemia (antibodies to lipoprotein lipase; systemic lupus erythematosus

Chronic idiopathic urticarial

Renal Disease

*These diseases are especially concerning in patients with preexisting hypertryglyceridemia, often on a genetic basis

+ Tryglycerides increase with each trimester, usually becomes symptomatic in 3rd trimester especially in susceptible patients

$\Psi$ carvedilol preferred in DM patients with hypertriglyceridemia if beta blockers are indicated

$\Delta$ in women who experienced hypertriglyceriglyceridemia with estrogen therapy

improved survival, tissue oxygenation, and microcirculatory perfusion, as well as less histopathologic damage in porcine model of severe acute pancreatitis [31].

\section{Target triglyceride level}

Triglycerides should be decreased and maintained at $<500 \mathrm{mg} / \mathrm{dL}$ to prevent progression to pancreatic necrosis and organ failure [2].

\section{Management strategies}

As of now, there are no definite guidelines for treating hypertriglyceride-induced pancreatitis but previous case reports and series have shown success with intravenous insulin and/or heparin [32-36], and plasmapheresis [28]. There are still no randomized studies that compare the efficacy of the different treatment regimens used in the management of this disease. Insulin may be considered the first choice with or without heparin in patients with concomitant hyperglycemia, but appears to be slower in action compared with apheresis, which decreases serum triglycerides and decrease symptoms in a very short period of time [34]. 


\section{Intravenous insulin \pm heparin}

Intravenous insulin and heparin administration should be considered in patients with concomitant hyperglycemia [32]. Insulin activate lipoprotein lipase which degrade chylomicrons into glycerol and free fatty acids resulting in rapid reduction of triglyceride levels [35,36]. Success has been reported using infusion of $5 \%$ dextrose with regular insulin (control serum glucose to $<200 \mathrm{mg} / \mathrm{dL}$ ) together with 5000 units of intravenous heparin twice daily in decreasing serum triglyceride levels to $<500 \mathrm{mg} / \mathrm{dL}$ and improve symptoms of pancreatitis within 4 days $[28,32]$. Subcutaneous regular insulin dosed at 0.1 unit $/ \mathrm{kg}$ decreases triglyceride levels within 4 hours but the effect is not sustainable long term [37]. Intravenous insulin is more efficacious than subcutaneous insulin for treating hypertriglycerideinduced pancreatitis.

While insulin has been shown to be effective when used as monotherapy [37], intravenous heparin does not. Heparin stimulates the release of endothelial lipoprotein lipase and causing an initial rise of the circulating enzyme but is immediately followed by its degradation in the liver resulting in its further depletion and recurrence of hypertriglyceridemia [38]. That is why heparin is recommended only as an adjunct treatment to insulin. A single bolus of low molecular weight heparin (Dalteparin) was found to deplete lipoprotein lipase similarly to unfractionated heparin [39].

\section{Apharesis}

Apheresis is capable of rapidly lowering markedly elevated triglyceride levels, clear prancreatic enzymes, and provides symptom relief from pancreatitis within 2.5 hours [40,41]. Reports have shown a $41 \%(1,406$ to $682 \mathrm{mg} / \mathrm{dL})$ decrease in serum triglycerides after 1 session of apheresis alone [42]. Several studies have shown that apheresis can significantly decrease serum triglycerides and cause both clinical and laboratory improvement when conservative treatment with diet and pharmaceutical drugs fail. [28, 43-45]. All these studies also stress the importance of performing apheresis as soon as possible to maximize therapeutic benefit. Its limiting factor is, however, its availability and very high cost.

\section{Prevention}

Prevention of initial and recurrent pancreatitis from hypertriglyceridemia should be emphasized. Data from NHANES (National Health and Nutrition Examination Survey) show that the prevalence of patients with very high triglycerides levels $(\geq 500 \mathrm{mg} /$ $\mathrm{dL}[\geq 5.65 \mathrm{mmol} / \mathrm{L}])$ are estimated to be to be $1.7 \%$ of the total study population or representing 3.4 million Americans [46]. The National Cholesterol Education Program Adult Treatment Panel III (ATP III) has already stated that the initial aim of therapy for patients is to prevent acute pancreatitis through triglyceride lowering [47]. Such therapy includes very low fat diets ( $\leq 15 \%$ of calorie intake), weight reduction and physical activity, and usually a triglyceride-lowering drug such as fibrates or nicotinic acid [30]. However, drug treatment (with fenofibrates or niacin) of asymptomatic persons with high triglyceride levels, particularly those less than $22.6 \mathrm{mmol} / \mathrm{L}$, is still not recommended because of lack of evidence for any considerable therapeutic benefit and subjects the patients to its adverse effects and high cost. [48]. Lastly, Pancreatic enzyme therapy has been shown to alleviate abdominal symptoms [49].

Risk factors for hypertriglyceridemia such as insulin resistance and alcoholism should be assessed in this setting as these are correctible factors that can be treated to avoid future bouts of pancreatitis in patients with occult hereditary lipid metabolism disorders. In such patients, providers should evaluate a patient's diet for contributors to elevated triglyceride levels. Epidemiologic reports have shown the glycemic index of foods has a direct correlation to triglyceride levels [50]. In addition, interventional studies have demonstrated that substituting foods possessing high glycemic indexes for foods with lower glycemic indexes can reduce a patient's serum triglyceride level by as much as $15 \%$ to $25 \%$ [51].

\section{Conclusion}

In conclusion, we provide an evidence based algorithm to summarize the approach to the etiology of hypertriglyceride-induced pancreatitis.

\section{References}

1. Fortson M, Freedman S, Webster PD 3rd (1995) Clinical assessment of hyperlipidemic pancreatitis. Am J Gastroenterol 90: 2134-2139.[Crossref]

2. Toskes PP (1990) Hyperlipidemic pancreatitis. Gastroenterol Clin North Am 19: 783791. [Crossref]

3. Sun L, Li W, Geng Y, Shen B, Li J (2011) Acute Pancreatitis in Pregnancy. Acta Obstetricia et Gynecologica Scandinavica. Nordic Federation of Societies of Obstetrics and Gynecology 90: 671-676. [Crossref]

4. Deng LH, Xue P, Xia Q, Yang X, Wan M (2008) Effect of admission hypertriglyceridemia on the episodes of severe acute pancreatitis. World J Gastroenterol 14: 4558-4561. [Crossref]

5. Dominguez-Munoz JE, Malfertheiner P, Ditschuneit HH, Blanco-Chavez J, Uhl W, et al. (1991) Hyperlipidemia in acute pancreatitis. Relationship with etiology, onset, and severity of the disease. Int J Pancreatol 10: 261-267. [Crossref]

6. Sandhu S, Al-Sarraf A, Taraboanta C, FrohlichJ, Francis GA (2011) Incidence of pancreatitis, secondary causes, and treatment of patients referred to a specialty lipid clinic with severe hypertriglyceridemia: a retrospective cohort study. Lipids in Health and Disease 10: 157 [Crossref]

7. Chang CC, Hsieh YY, Tsai HD, et al. (1998) Acute pancreatitis in pregnancy Zhonghua yi xue za zhi-Chinese Medical Journal; Free China ed 61: 85-92. [Crossref]

8. O’Brien T, Dinneen SF, O' Brien PC, Palumbo PJ (1993) Hyperlipidemia in patient with primary and secondary hypothyroidism. Mayo Clin Proc 68: 860-866. [Crossref]

9. Durrington P (2003) Dyslipidaemia. Lancet 362: 717-731. [Crossref]

10. Fallat RW, Vester JW, Glueck CJ (1973) Suppression of amylase activity by hypertriglyceridemia. JAMA 225: 1331-1334. [Crossref]

11. Warshaw AL, Bellini CA, Lesser PB (1975) Inhibition of serum and urine amylase activity in pancreatitis with hyperlipemia. Ann Surg 182: 72-75. [Crossref]

12. Zhang W, Zhao Y, Zeng Y, Yu X, Yao J, et al. (2012) Hyperlipidemic Versus NormalLipid Acute Necrotic Pancreatitis: Proteomic Analysis Using an Animal Model. Pancreas 41: 317-322. [Crossref]

13. Vonlaufen A, Wilson JS, Apte MV (2008) Molecular mechanisms of pancreatitis: current opinion. J Gastroenterol Hepatol 23: 1339-1348. [Crossref]

14. Saharia P, Margolis S, Zuidema GD, Cameron JL (1977) Acute pancreatitis with hyperlipemia: studies with an isolated perfused canine pancreas. Surgery 82: 60-67. [Crossref]

15. Qihui C, Xiping Z, Xianfeng D (2012) Clinical study on acute pancreatitis in pregnancy in 26 cases. Gastroenterol Res Pract 2012: 271925. [Crossref]

16. Nair S, Yadav D, Pitchumoni CS (2000) Association of diabetic ketoacidosis and acute pancreatitis: observations in 100 consecutive episodes of DKA. Am J Gastroenterol 95: 2795-2800. [Crossref]

17. Brunzell JD (1999) Familial lipoprotein lipase deficiency. GeneReviews ${ }^{\mathrm{TM}}$ [Internet] [Crossref]

18. Coca-Prieto I, Valdivielso P, Olivecrona G, Ariza M, Rioja J, et al.(2009) Lipoprotein lipase activity and mass, apolipoprotein C-II mass and polymorphisms of apolipoproteins $\mathrm{E}$ and $\mathrm{A} 5$ in subjects with prior acute hypertriglyceridaemic pancreatitis. BMC Gastroenterol 9: 46. [Crossref] 
19. Pownall HJ, Ballantyne CM, Kimball KT, Simpson SL, Yeshurun D, et al. (1999) Effect of moderate alcohol consumption on hypertriglyceridemia: a study in the fasting state. Arch Intern Med 159: 981-987. [Crossref]

20. Goldenberg NM, Wang P, Glueck CJ (2003) An observational study of severe hypertriglyceridemia, hypertriglyceridemic acute pancreatitis, and failure of triglyceride-lowering therapy when estrogens are given to women with and without familial hypertriglyceridemia. Clin Chim Acta 332: 11-9. [Crossref]

21. Fredrickson DS (1971) An international classification of hyperlipidemias and hyperlipoproteinemias. Ann Intern Med 75: 471-472. [Crossref]

22. Taghibiglou C, Carpentier A, Van Iderstine S, Chen B, Rudy D, Aiton A, et al. (2000) Mechanisms of hepatic very low density lipoprotein overproduction in insulin resistance. Evidence for enhanced lipoprotein assembly, reduced intracellular ApoB degradation, and increased microsomal triglyceride transfer protein in a fructose-fed hamster model. J Biol Chem: 275: 8416-8425. [Crossref]

23. Ginsberg HN, Zhang YL, Hernandez-Ono A (2005) Regulation of plasma triglycerides in insulin resistance and diabetes. Arch Med Res 36: 232-240. [Crossref]

24. Ilag, LL (2001) Case Study: Hypertriglyceridemia in a woman with insulin resistance. doi: 10.2337/diaclin.19.4.183 Clinical Diabetes 19: 183-184.

25. Brunzell JD, Porte D Jr, Bierman EL (1979) Abnormal lipoprotein lipase mediated plasma triglyceride removal in untreated diabetes mellitus associated with hypertriglyceridemia. Metabolism. 28: 901-907.

26. Fulop M, Eder HA (1989) Plasma triglycerides and cholesterol in diabetic ketosis. Arch Intern Med 149: 1997-2002. [Crossref]

27. Van de Wiel A (2012) The effect of alcohol on postprandial and fasting triglycerides. Int J Vasc Med: 862504. [ Crossref]

28. Schneider J, Liesenfeld A, Mordasini R, Schubotz R, Zöfel P, et al. (1985) Lipoprotein fractions, lipoprotein lipase and hepatic triglyceride lipase during short-term and longterm uptake of ethanol in healthy subjects. Atherosclerosis.57: 281-291.

29. Brunzell JD (2007) Clinical practice. Hypertriglyceridemia. N Engl J Med 357: 10091017. [Crossref]

30. Kyriakidis AV, Karydakis P, Neofytou N, Pyrgioti M, Vasilakakis D, et al. (2005) Plasmapheresis in the management of acute severe hyperlipidemic pancreatitis: report of 5 cases. Pancreatology 5: 201-204. [Crossref]

31. Keech A, Simes RJ, Barter P, Best J, Scott R, Taskinen MR, et al. (2005) FIELD study investigators. Effects of long-term fenofibrate therapy on cardiovascular events in 9795 people with type 2 diabetes mellitus (the FIELD study): randomised controlled trial. Lancet 366: 1849-1861. [Crossref]

32. Miller M, Stone NJ, Ballantyne C, Bittner V, Criqui MH, Ginsberg HN, et al. (2011) American Heart Association Clinical Lipidology, Thrombosis, and Prevention Committee of the Council on Nutrition, Physical Activity, Metabolism. Triglycerides and cardiovascular disease: a scientific statement from the American Heart Association. Circulation 123: 2292-2333. [Crossref]

33. Trepte CJ, Bachmann KA, Stork JH, Friedheim TJ, Hinsch A, et al. (2013) The impact of early goal-directed fluid management on survival in an experimental model of severe acute pancreatitis. Intensive Care Med 39: 717-726. [Crossref]

34. Alagözlü H, Cindoruk M, Karakan T, Unal S (2006) Heparin and insulin in the treatment of hypertriglyceridemia-induced severe acute pancreatitis. Dig Dis Sci 51: 931-933. [Crossref]

35. Jain P, Rai RR, Udawat H, Nijhawan S, Mathur A (2007) Insulin and heparin in treatment of hypertriglyceridemia-induced pancreatitis. World J Gastroenterol 13: 2642-2643. [Crossref]
36. Bota VM (2013) Urgent apheresis combined with insulin infusion in hypertriglyceridemia-induced acute pancreatitis. The American Journal of Emergency Medicine 31: 452.e1-452.e2. [ Crossref]

37. Eckel RH (1989) Lipoprotein lipase. A multifunctional enzyme relevant to common metabolic diseases. N Engl J Med 320: 1060-1068. [ Crossref]

38. KORN ED (1955) Clearing factor, a heparin-activated lipoprotein lipase. I. Isolation and characterization of the enzyme from normal rat heart. J Biol Chem 215: 1-14. [Crossref]

39. Jabbar MA, Zuhri-Yafi MI, Larrea J (1998) Insulin therapy for a non-diabetic patien with severe hypertriglyceridemia. J Am Coll Nutr 17: 458-461. [Crossref]

40. Weintraub M, Rassin T, Eisenberg S, Ringel Y, Grosskopf I, Iaina A, et al. (1994) Continuous intravenous heparin administration in humans causes a decrease in serum lipolytic activity and accumulation of chylomicrons in circulation. J Lipid Res 35: 229238. [Crossref]

41. Asström B, Stegmayr B, Gupta J, Olivecrona G, Olivecrona T (2005) A single bolus of a low molecular weight heparin to patients on haemodialysis depletes lipoprotein lipase stores and retards triglyceride clearing. Nephrol Dial Transplant 20: 1172-1179.

42. Tsuang W, Navaneethan U, Ruiz L, Palascak JB, Gelrud A (2009) Hypertriglyceridemic pancreatitis: presentation and management. Am J Gastroenterol 104: 984-991. [Crossref]

43. Piolot A, Nadler F, Cavallero E, Coquard J, Jacotot B (1996) Prevention of recurren acute pancreatitis in patients with severe hypertriglyceridemia: value of regular plasmapheresis. Pancreas 13: 96-99. [Crossref]

44. Kadikoylu G, Yavasoglu I, Bolaman Z (2006) Plasma exchange in severe hypertriglyceridemia a clinical study. Transfus Apher Sci 34: 253-257. [Crossref]

45. Stefanutti C, Di Giacomo S, Vivenzio A, Labbadia G, Mazza F, et al. (2009). Therapeutic plasma exchange in patients with severe hypertriglyceridemia: a multicenter study. Artif Organs 33: 1096-1102. [Crossref]

46. Lennertz A, Parhofer KG, Samtleben W, Bosch T (1999) Therapeutic plasma exchange in patients with chylomicronemia syndrome complicated by acute pancreatitis. Ther Apher 3: 227-233. [Crossref]

47. Yeh JH, Chen JH, Chiu HC (2003) Plasmapheresis for hyperlipidemic pancreatitis. $J$ Clin Apher 18: 181-185. [Crossref]

48. Christian JB, Bourgeois N, Snipes R, Lowe KA (2011) Prevalence of severe (500 to 2,000 mg/dl) hypertriglyceridemia in United States adults. Am J Cardiol 107: 891-897. [Crossref]

49. National Cholesterol Education Program (2002) Expert Panel on Detection, Evaluation, and Treatment of High Blood Cholesterol in Adults (Adult Treatment Panel III). Circulation 106: 3143-3421.

50. Lederle FA, Bloomfield HE (2012) Drug treatment of asymptomatic hypertriglyceridemia to prevent pancreatitis: where is the evidence? Ann Intern Med 157: 662-664. [Crossref]

51. Pelkman CL (2001) Effects of the glycemic index of foods on serum concentrations of high-density lipoprotein cholesterol and triglycerides. Curr Atheroscler Rep 3: 456461. [Crossref]

52. Yadav D, Pitchumoni CS (2003) Issues in hyperlipidemic pancreatitis. $J$ Clin Gastroenterol 36: 54-62. [Crossref]

53. Jain P, Rai RR, Udawat H, Nijhawan S, Mathur A (2007) Insulin and heparin in treatment of hypertriglyceridemia-induced pancreatitis. World J Gastroenterol 13 : 2642-2643. [Crossref]

Copyright: (C2016 Lu MLRY. This is an open-access article distributed under the terms of the Creative Commons Attribution License, which permits unrestricted use, distribution, and reproduction in any medium, provided the original author and source are credited. 